\title{
PAPR reduction by pulse shaping using Nyquist linear combination pulses
}

\author{
Cesar A. Azurdia-Meza, Kyujin Lee, and Kyesan Lee ${ }^{a}$ ) \\ Department of Electronics and Radio Engineering, Kyung Hee University, \\ 1 Seocheon-dong, Giheung-gu, Yongin-si, Gyeonggi-do 446-701, Republic of Korea \\ a)kyesan@khu.ac.kr
}

\begin{abstract}
Peak-to-average power ratio (PAPR) plays an important role in the design of wireless communication systems because a high PAPR decreases the efficiency of the power amplifier. A new family of Nyquist pulses was derived by applying a linear combination between two Nyquist pulses. The proposed Nyquist pulses are utilized for pulse shaping to reduce PAPR. The investigated pulses are characterized by a new design parameter $(p)$, giving an additional degree of freedom to minimize PAPR for a given roll-off factor $\alpha$. While keeping the same bandwidth, the frequency responses of the proposed filters are unique with different values of the parameter $p$. Simulations showed that the optimum proposed pulse shaping filters provide better performance in PAPR reduction compared to those of the RC filter and other existing filters evaluated in various systems.
\end{abstract}

Keywords: Nyquist's first criterion, peak-to-average power ratio (PAPR), power amplifier

Classification: Microwave and millimeter wave devices, circuits, and systems

\section{References}

[1] J. Choi, D. Kang, D. Kim, J. Park, B. Jin, and B. Kim, "Power Amplifiers and Transmitters for Next Generation Mobile Handsets," J. Semiconductor Technology and Science, vol. 9, no. 4, pp. 249-256, Dec. 2009.

[2] H. G. Myung, J. Lim, and D. J. Goodman, "Single carrier FDMA for uplink wireless transmission," IEEE Veh. Technol. Mag., vol. 1, no. 3, pp. 30-38, Sept. 2006.

[3] H. G. Myung, J. Lim, and D. J. Goodman, "Peak-to-average power ratio of single carrier FDMA with pulse shaping," Proc. IEEE 17th Int. Symp. PIMRC, Helsinki, Finland, pp. 1-5, Sept. 2006.

[4] B. Berglund, J. Johansson, and T. Lejon, "High efficiency power amplifiers," Ericsson Review, vol. 83, no. 3, pp. 92-96, 2006.

[5] L. Lei, S. Enchang, and Z. Yanhua, "A Method for PAPR Reduction Using Pilot Sequences in SC-FDMA," Proc. 6th Int. Conf. WiCom, Chengdu, China, pp. 1-6, Sept. 2010.

[6] R. V. Nee and R. Prasad, OFDM for Wireless Multimedia Communication, Artech House, Boston, 2000. 
[7] G. Huang, A. Nix, and S. Armour, "Impact of Radio Resource Allocation and Pulse Shaping on PAPR of SC-FDMA Signals," Proc. IEEE 18th Int. Symp. PIMRC, Athens, Greece, pp. 1-5, Sept. 2007.

[8] B. Farhang-Boroujeny, "A Square-Root Nyquist (M) Filter Design for Digital Communication Systems," IEEE Trans. Signal Process., vol. 56, no. 5, pp. 2127-2132, May 2008.

[9] P. S. Rha and S. Hsu, "Peak-to-average power ratio (PAPR) by Pulse Shaping using a New Family of Generalized Raised Cosine Filters," Proc. IEEE 58th Vehicular Technology Conference, Orlando, USA, pp. 706710, Oct. 2003.

[10] Y. D. Wei and Y. F. Chen, "Peak-to-average power ratio (PAPR) reduction by pulse shaping using the K-exponential filter," IEICE Trans. Commun., vol. E93-B, no. 11, pp. 3180-3183, Nov. 2010.

[11] J. O. Scanlan, "Pulses Satisfying the Nyquist Criterion," IET Electronics Lett., vol. 28, no. 1, pp. 50-52, Jan. 1992.

[12] N. C. Beaulieu and M. O. Damen, "Parametric construction of NyquistI pulses," IEEE Trans. Commun., vol. 52, no. 12, pp. 2134-2142, Dec. 2004.

[13] H. Nyquist, "Certain topics in telegraph transmission theory," AIEE Trans., vol. 47, pp. 617-644, 1928.

[14] P. Sandeep, S. Chandan, and A. K. Chaturvedi, "ISI-Free Pulses with Reduced Sensitivity to Timing Errors," IEEE Commun. Lett., vol. 9, no. 4, pp. 292-294, April 2005.

[15] G. Strang, Introduction to Linear Algebra, Wellesley-Cambridge Press, Wellesley, 1998.

[16] B. Chatelain and F. Gagnon, "Peak-to-average power ratio and intersymbol interference reduction by Nyquist optimization," Proc. 60th IEEE Vehicular Technology Conference, Los Angeles, USA, pp. 954-958, Sept. 2004.

\section{Introduction}

New wireless communication standards, such as 3GPP long-term evolution (LTE), employ orthogonal frequency-division multiplexing (OFDM) based techniques for spectral efficiency. Despite the benefits offer by the newly introduced standards, the power amplifier of the mobile terminal has to accommodate higher peak-to-average power ratios (PAPR) than prior standards, such as WCDMA, CDMA, and EDGE [1]. As a result, PAPR plays an important role in the design of wireless communication systems because power amplifiers are usually operated in the linear mode rather than the saturation mode. A bandlimited signal with high PAPR requires a large back-off to guarantee that the power amplifier operates inside its linear region in order not to distort the transmitted signals $[2,3]$. In addition, signals with high PAPR require higher dynamic ranges of digital-to-analog converters (DAC) at the power amplifier.

Research has been ongoing the last several years trying to design a power amplifier that maintains high efficiency for signals with high PAPR. Several power amplifier modulation technologies have the potential to work with high PAPR signals, such as Doherty amplifiers $[1,4]$, but at the moment, these 
technologies are at an experimental stage. Therefore, several methods for reducing PAPR in OFDM based systems have been developed; such as probabilistic algorithms [5], non-linear expanding and compressing methods [5], and pre-coding methods [6].

Prior to transmitting the modulated symbols, pulse shaping is required to bandlimit the transmitted signal in OFDM-based systems [2, 7]. However, pulse shaping to limit the frequency bandwidth enlarges the PAPR of the transmitted signals, resulting in a trade-off between PAPR and bandwidth reduction. Research has been ongoing over the last years trying to design a pulse shaping filter that limits PAPR without degrading the system's performance, based on the knowledge that, and compared to other PAPR reduction techniques, pulse shaping is an effective and simple method $[3,8,9,10]$.

In [9], a new family of generalized raised cosine (RC) filters was derived to reduce PAPR. Recently, a new family of pulse shaping filters, known as the K-exponential filters [10], was proposed to reduce PAPR. Both of these families of filters hold an additional design parameter, giving an additional degree of freedom for minimizing PAPR for a given roll-off factor, but are characterized by complex impulse response expressions.

In this letter, a linear combination between two Nyquist pulses was proposed. The traditional RC pulse and the parametric linear pulse (PLP) proposed in [11], and later derived in [12], were linearly combined. The proposed family of pulses has an additional parameter, giving an additional degree of freedom to minimize PAPR for a fixed roll-off factor. The proposed pulse shaping filter has a simpler impulse response expression compared to those of the existing filters in [9] and [10]. OFDM-based systems were employed to demonstrate the PAPR performance improvement.

\section{Nyquist linear combination pulses}

Nyquist's first criterion for distortionless transmissions within a bandlimited channel is defined as follows [13]

$$
h(n T)=\left\{\begin{array}{ll}
1, & n=0 \\
0, & n= \pm 1, \pm 2, \pm 3, \ldots
\end{array},\right.
$$

where $h(t)$ is the impulse response and $T$ is the symbol period. This criterion guarantees that a sequence of pulses, sampled at the optimum and uniformly spaced sampling instants, will have zero intersymbol interference (ISI).

According to [14], a linear combination between two pulses that comply with (1) guarantees that the resulting pulse will also be Nyquist. We considered the linear combination between the RC pulse and the PLP pulse as follows

$$
h(t)=p h(t)_{P L P}+(1-p) h(t)_{R C},
$$

where $p$ is the constant that corresponds to the linear combination and it is defined for all real numbers [15]. The introduced linear combination constant adds an additional degree of freedom to minimize PAPR for a given roll-off factor, because to reduce PAPR at the transmitter side, we need to design a 
pulse shaping filter with reduced tail size $[8,16]$. The impulse responses of the PLP and RC pulses are given in [12], and are inserted into (2) to obtain the impulse response of the proposed Nyquist linear combination pulse (NLCP) as follows

$$
h(t)_{N L C P}=p\left[\frac{\sin (\pi \tau) \sin (\alpha \pi \tau)}{\alpha \pi^{2} \tau^{2}}\right]+(1-p)\left[\frac{\sin (\pi \tau) \cos (\alpha \pi \tau)}{\pi \tau\left(1-4 \alpha^{2} \tau^{2}\right)}\right],
$$

where $\tau$ is the normalized time $(\tau=t / T), T$ is the symbol period, and $\alpha$ is the roll-off factor $(0 \leq \alpha \leq 1)$. In the rest of this letter we will express the time-domain functions in terms of the normalized time for simplicity. After some algebraic manipulations, the impulse response of the NLCP is given as follows

$$
h(t)_{N L C P}=\frac{\sin (\pi \tau)}{\pi \tau}\left[\frac{p \sin (\alpha \pi \tau)}{\alpha \pi \tau}+\frac{(p-1) \cos (\alpha \pi \tau)}{4 \alpha^{2} \tau^{2}-1}\right] .
$$

The proposed pulse in (4), evaluated for $t=0$, and for any value of $p$, is always equal to one, according to Nyquist's first criterion [11, 12, 13]. The frequency response of the proposed pulse can be evaluated through a numerical Fourier transform.

The time and frequency responses of the proposed filter, as well as for the RC filter, are shown in Figs. 1 and 2, respectively, for a roll-off factor $\alpha$ and different values of $p$. Regarding the impulse response of the NLCP for different values of $p$, and related to the mainlobe, the $\mathrm{RC}$ filter has larger sidelobes than those of the proposed filter with $p=0.5, p=1.5$, and $p=2.5$ as examples. From examination of Fig. 1, it can be inferred that by selecting the proper value of $p$, smaller relative magnitudes in the two largest sidelobes can be obtained. And according to $[8,10,16]$, PAPR reduction is achieved by minimizing the amplitudes of the two largest sidelobes. The proposed family

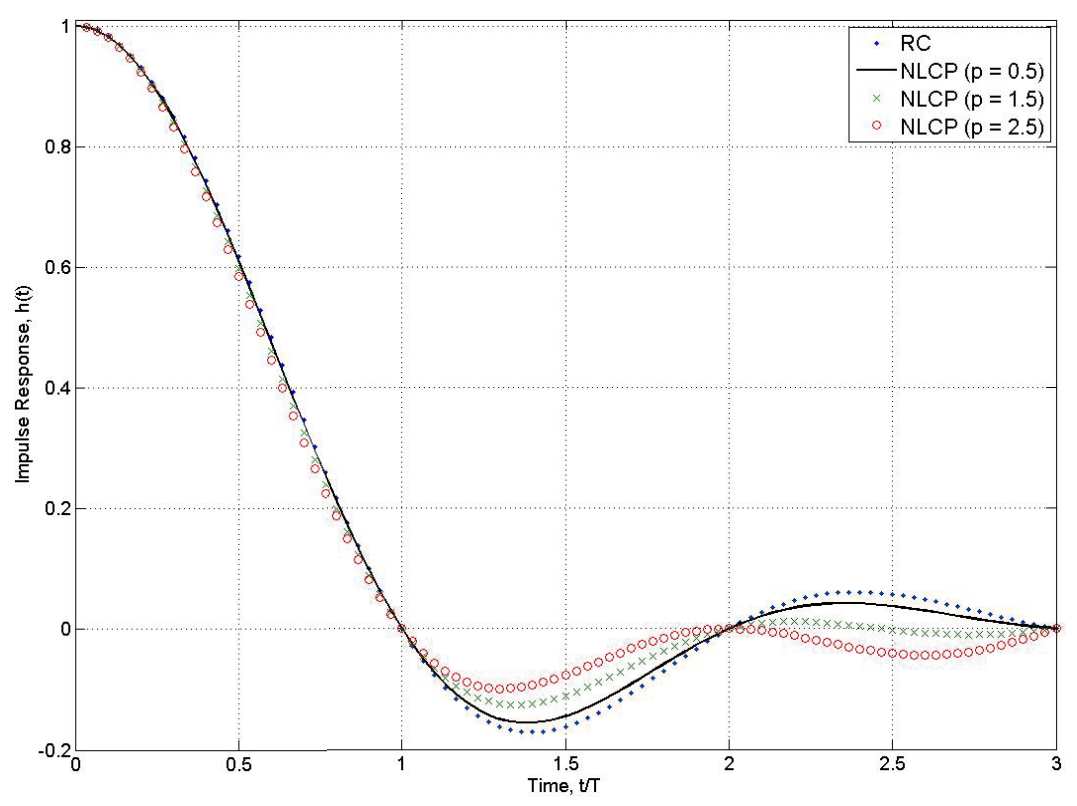

Fig. 1. Impulse response of the RC pulse and the NLCP for different values of $p$ and $\alpha=0.35$. 


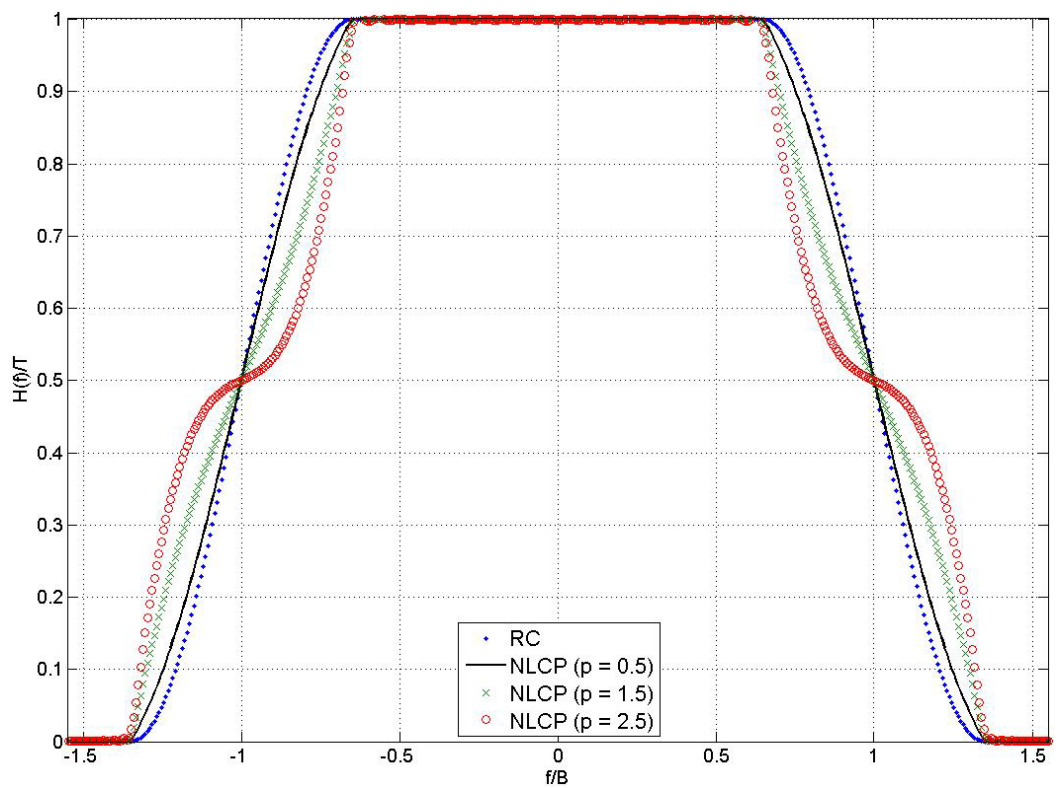

Fig. 2. Frequency response of the RC pulse and the NLCP for different values of $p$ and $\alpha=0.35$.

of pulses maintains the same bandwidth compared to that of the $\mathrm{RC}$ pulse for a roll-off factor $\alpha$ and different values of $p$; therefore does not introduce additional out-of band radiation into the transmitted signals, which is a major concern in the design of pulse shaping filters.

\section{Performance evaluation}

In the 3GPP LTE standard, the technologies being implemented for downlink and uplink consist of OFDMA and SC-FDMA, respectively [2, 3]. Both techniques are based on the OFDM technology. To validate the efficacy of the proposed pulse shaping filters for PAPR reduction, the interleaved frequency division multiple access (IFDMA) mode of SC-FDMA and traditional OFDM systems were implemented.

The total PAPR at the transmitter side is determined by the combination of the technology scheme and the pulse shaping filter implemented. To evaluate the PAPR of individual system configurations, we simulated the transmission of $10^{5}$ system blocks for SC-IFDMA and $10^{6}$ system blocks for OFDM. After calculating the PAPR of each block, the data was presented as an empirical complementary cumulative distribution function (CCDF) [6]. As it was done in [10], PAPR was measured and analyzed on the spectrum of the proposed NLCP and the other filters implemented for comparison. Table I illustrates the parameters implemented in our simulations. The parameters applied in our simulations agree with the ones in [10] for comparison purposes. In all of our simulations we used a roll-off factor of 0.35 , which is a typical value used in literature.

The optimal value of the linear combination constant $p$ for PAPR reduction was selected through extensive computer simulations because an analytical solution for the optimal $p$ seems unrealizable. The same approach 
Table I. Simulation Parameters.

\begin{tabular}{c|c}
\hline Parameter & Value \\
\hline \hline Technology & SC-IFDMA/OFDM \\
\hline Modulation & 16QAM \\
\hline Transmission Bandwidth & $20 \mathrm{MHz}$ \\
\hline Number of Subcarriers & 256 \\
\hline Input Data Block Size & 64 \\
\hline Uniform Random Data Points & $10^{5} / 10^{6}$ \\
\hline Roll-off Factor & 0.35 \\
\hline Block Oversampling & 4 \\
\hline
\end{tabular}

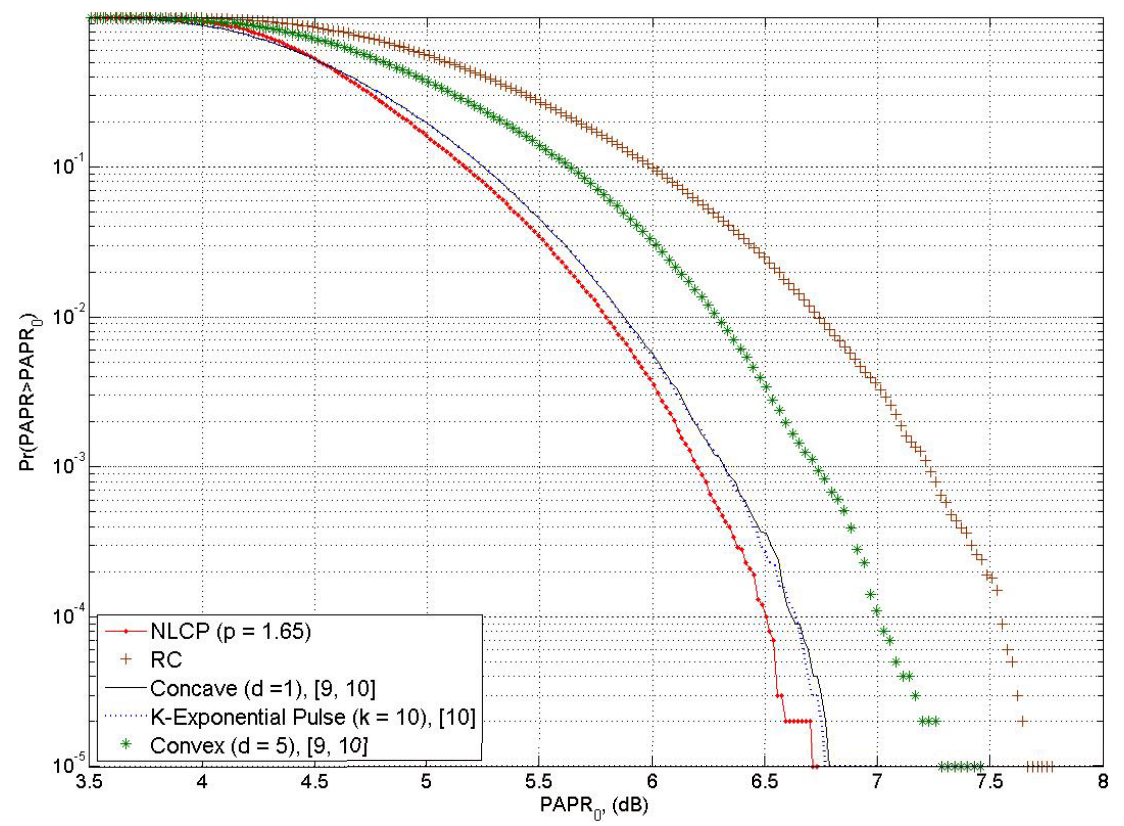

Fig. 3. CCDF of PAPR for a SC-IFDMA system with $\alpha=0.35$ and 16QAM.

was taken in [10] for selecting the optimum $k$, as well as for the optimum $d$ proposed for the pulses described in [9]. The theoretical derivation of the optimum $p$ will be investigated in future studies.

In Fig. 3 we demonstrate the effectiveness of the proposed pulse shaping filter for the interleaved mode of the SC-FDMA system. It was found through extensive computer simulations that the optimum NLCP uses a linear combination constant equal to 1.65. The proposed optimum NLCP has the lowest PAPR among the evaluated pulses for comparison. The reasons in PAPR reduction are explained as follows. The optimum proposed pulse has smaller relative magnitudes in its two largest sidelobes compared to those of the pulses implemented for comparison. As a result, and according to $[8,10,16]$, a pulse with smaller sidelobes would lead to a smaller PAPR.

The performance of the proposed pulse shaping filter for OFDM is shown in Fig. 4. The OFDM system was evaluated using $10^{6}$ system blocks to obtain smooth curves and to have more data points in order to have reliability in the proposed system. For the sake of clarity, only the optimum proposed pulse, the RC filter and the optimum K-exponential filter [10] were evaluated. The previous argument is not a major concern because in [10], the optimum K- 


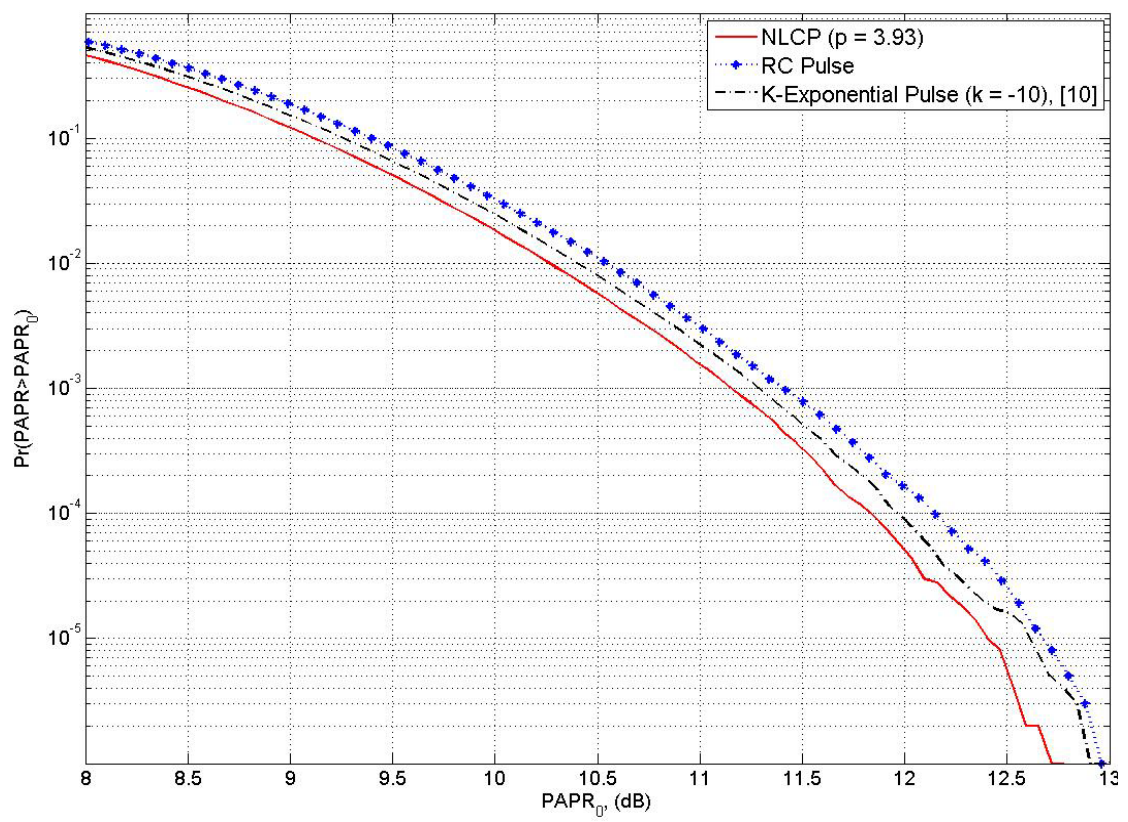

Fig. 4. CCDF of PAPR for an OFDM system with $\alpha=$ 0.35 and 16QAM.

exponential pulse has already been shown to outperform the optimum filters proposed in [9]. It was found through extensive computer simulations that the optimum NLCP linear combination constant for PAPR reduction in OFDM systems is equal to 3.93. Even though the PAPR performance improvement is not so significant for the OFDM-based system, the proposed optimum pulse outperforms the filters for comparison. As in [10], the optimum linear combination constant varies according to the technology implemented.

Despite that the optimum filters proposed in [9] and [10] also have an additional design parameter, the optimum NLCP pulses have superior performance in PAPR reduction. Our novel family of pulses has a simpler impulse response expression in comparison to the prior pulses. This is extremely important because OFDM-based systems use an inverse discrete Fourier transform (IDFT) for transforming the subcarrier amplitudes into a complex time domain signal. Afterwards, a convolution between the complex time domain signal and the impulse response takes place. As a result, the complexity of the convolution between the modulated signals and our proposed optimum filter is reduced when compared to that of the other evaluated pulses. Therefore, less complex digital systems will be needed by implementing our proposed optimum pulses.

\section{Conclusion}

In this letter, a novel family of Nyquist pulses has been derived by implementing a linear combination between the RC and the PLP pulse. The new family of pulses is characterized by an additional design parameter $p$, which gives an additional degree of freedom to minimize PAPR for a given roll-off factor $\alpha$ and transmission scheme. The optimum values of $p$ were found by 
using extensive computer simulations, and were found equal to 1.65 for the SC-IFDMA scheme and 3.93 for the OFDM system. Simulations showed that the optimum proposed filters provide better performance regarding PAPR reduction compared to that of the existing filters described in [9] and [10]. The proposed pulse shaping filters have a much simpler impulse response compared to those of the prior filters; therefore, adding flexibility and simplicity into the system.

\section{Acknowledgments}

This work was supported by the IT R\&D program of MKE/KEIT [10035362, Development of Home Network Technology based on LED-ID]. 